\title{
Morteros a base de suelos. Caracterización
}

\author{
JORGE LUIS A. CABRERA, Msc.Ing.*; YISEL FERNÁNDEZ COLLAZO, Ing.**; \\ NIURVIS GONZÁLEZ RAMÍREZ, Ing** ySANTIAGO BERMÚDEZ VALLES, Tec.* \\ ${ }^{(*)}$ Centro Técnico para el Desarrollo de los Materiales de Construcción; ${ }^{(* *)}$ Instituto Superior Superior \\ Politécnico José Antonio Echevarría (ISPJAE)
}

Fecha de recepción: $22-I V-99$

\begin{abstract}
RESUMEN
Los objetivos planteados en este trabajo se enmarcan en un estudio con diferentes dosificaciones volumétricas, empleando varios tipos de suelos como agregados y cemento e hidrato de cal como aglomerante para ser empleados en morteros de albañilería.
\end{abstract}

Los morteros fueron caracterizados, determinándose la $R$ a/c, $R f$ y $R c$ a diferentes edades, $R$ adh. a 28 dias, absorción capilar hasta valores constantes y coste/ $\mathrm{m}^{3}$ de morteros.

Los resultados demuestran que los morteros de albañilería confeccionados con suelos cumplen con los parámetros necesarios para ser empleados en obras de construcción con suelo-cemento.

\section{SUMMARY}

The goals states in this work are included in a study with differents volumetric dosifications, ussing different kinds of soils like agregates, and cement and lime like binding material to be used in mansoniry mortars.

The mortars were caracterized by determining the $R a / c, R f$ and $R c$ to different ages, $R$ adh. to 28 days, capilar absorption till constant values and cost $/ \mathrm{m}^{3}$ of mortars.

The results show that mansonry mortars made with soils fulfil the necessary parameters to be used in construction works with cement-soils.

\section{INTRODUCCIÓN}

La tierra es un material de construcción que, por su extraordinaria abundancia (el 74\% de la corteza terrestre es tierra: arcilla y lateritas fundamentalmente), hace que no sea factible ni como mercadería ni como monopolio (1). Se conoce de construcciones con tierra (obras defensivas y viviendas) desde los siglos VII y VI A.N.E. en Italia y algunas zonas de la gran Muralla China, pero incluso antes, por los años 3.100 A.N.E. nos encontramos con las ruinas de Tepe Jahya en la zona del sureste de Irán, lo cual es hoy un montículo de $18 \mathrm{~m}$ de altura y $180 \mathrm{~m}$ de diámetro (2) por lo tanto, la tierra ha sido y nadie lo pone en duda, un excelente material para la construcción.. La tierra fue el material con que se construyeron ciudades como Igoubmime en el Valle del Rheoris en Marruecos; murallas como las de Chan Chan en Perú; templos como el de Pueblo de la isla de Nuevo México (EE UU ); o la gran mezquita de Djenné en Malí; humildes construcciones rurales en el Dauphiné francés o viviendas burguesas en la Guinea colonial (1).

Según Salas (1) en 1986 vivían, se alojaban o simplemente se protegían de la intemperie mediante construcciones con tierra, un tercio de la humanidad, o sea, 1500 millones de seres humanos.

Con el desarrollo de la sociedad y el descubrimiento de un número significativo de materiales para la construcción, la tierra pasa a ocupar un lugar remoto en las construcciones y sólo se conserva su empleo en aquéllos lugares en que, por necesidades económicas, no hay posibilidad del empleo de otro tipo de material. Incluso hoy en día se asocia el uso de la tierra en las construccio- 
nes como sinónimo de subdesarrollo, pobreza, etc., lo cual no es exactamente así, ya que la tierra sigue siendo un material de construcción, como dijo Salas (1) . mientras existan esos cien millones de seres humanos sin ningún tipo de cobijo y más de mil millones viviendo en chabolas, ranchitos, convertillos, pueblos jóvenes, favelas y callampas

Afortunadamente, en las últimas décadas se ha incrementado el interés por el estudio de la tierra como material para la construcción y así tenemos que en Grenoble (CRATERRE)); en París (C.S.T.B.); en Lyon (INJA) ; en Bélgica, Alemania, Estados Unidos Perú y Brasil, entre otros, están investigando el uso de la tierra. También la RILEM creó el Comité Técnico para los materiales de construcción a base de tierra y en Francia hay más de 20 instituciones y escuelas técnicas dedicadas al estudio de la tierra. Más reciente, con la aparición del cemento portland, comienza el empleo de los suelos mezclados con éste en subbase de pavimentos y, más tarde (mediados de la década del 70), en la construcción experimental de vivienda. En estas construcciones, el suelo-cemento se empleó fundamentalmente en la conformación de ladrillos y bloques.

Por todo lo anteriormente expuesto, se plantea la realización de este trabajo, cuyo objetivo es la caracterización de morteros elaborados con cemento e hidrato de cal como aglomerantes y diferentes suelos como agregado.

\section{METODOLOGÍA APLICADA AL ESTUDIO EXPERIMENTAL}

\subsection{Materiales y Dosificaciones}

Los materiales aglomerantes empleados fueron cemento portland P-350, portland puzolánico PP-250 e hidrato de cal (DPA). El DPA es un hidrato de cal obtenido como subproducto en la producción del acetileno.

Los suelos fueron clasificados, según el sistema de clasificación H.B.R. (Highway Research Board) como se muestra en la Tabla 1. Todos los suelos fueron pasados por el tamiz de 2,31 mm de abertura.

Las dosificaciones empleadas se muestran en la Tabla 2.

\subsection{Métodos de ensayo}

Los morteros fueron dosificados en proporciones volumétricas y homogeneizadas y preparadas en una mezcladora, empleándose el agua necesaria para alcanzar una fluidez en la mesa de sacudidas de $110 \pm 10 \mathrm{~mm}$. Este parámetro de trabajabilidad se mantuvo fijo para todas las dosificaciones.

Los morteros de albañilería se confeccionaron en moldes de $40 \times 40 \times 160 \mathrm{~mm}$, empleando la metodología que aparece en la NC.52-79:93 (3).

\section{Ensayo de resistencia a flexión y compresión}

Se realizaron ensayos de resistencia a flexión y compresión a las edades de 7, 28, 90, 210 y 365 días para ver el comportamiento que presentan dichos morteros a través del tiempo. Las probetas fueron ensayadas de acuerdo a la Norma de Ensayos Físico-Mecánicos de Cementos NC.54-207:80 (4), por no existir una norma específica para este tipo de morteros.

\section{Ensayo de adherencia a tracción}

El ensayo se realizó empleando la metodología descrita en (5). Como substrato se emplearon ladrillos macizos de suelo-cemento, los cuales fueron humedecidos

TABLA 1

Características de los suelos

\begin{tabular}{|c|c|c|}
\hline TIPODE SUELO & CLASIFICACIÓN & OBSERVACIONES \\
\hline A & A-1-a & $\begin{array}{c}\text { Suelo gravoso-arenoso con pequeñas cantidades } \\
\text { de limo }\end{array}$ \\
\hline B & A-1-b & $\begin{array}{c}\text { Suelo gravoso-arenoso con pequeñas cantidades } \\
\text { de limo }\end{array}$ \\
\hline C & A-4 & $\begin{array}{c}\text { Suelo que presenta gravas y arena, pero con alto } \\
\text { contenido en limo (18\%) }\end{array}$ \\
\hline D & A-7-5 & $\begin{array}{c}\text { Suelo muy fino donde predominan los materiales } \\
\text { arcillosos }\end{array}$ \\
\hline
\end{tabular}


TABLA 2

Dosificaciones Volumétricas

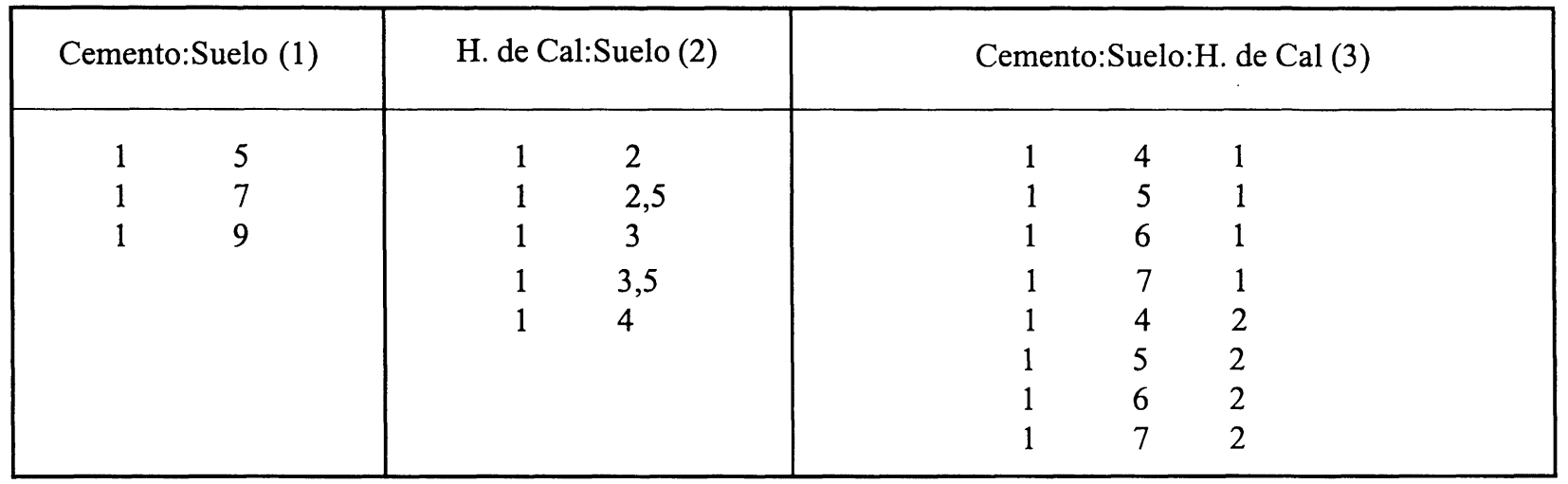

(1) Se emplearon con todos los suelos

$(2,3)$ Sólo con el suelo D, debido a que como es un suelo arcilloso está recomendado para trabajarlo con hidrato de cal.

previamente antes de la colocación del mortero. El arrancamiento de las chapillas se realizó a los 28 días de curado.

\section{Ensayo de absorción capilar}

Las probetas preparadas según NC.52-79:93 fueron puestas en la sala de ensayo a temperatura ambiente durante 6 días y luego colocadas en posición vertical sobre un lecho de arena fina sumergidas en una pequeña capa de agua de aproximadamente $5 \mathrm{~mm}$. Se evaluó la variación de peso a las edades de ensayo, determinándose la absorción en $\mathrm{g} / \mathrm{cm}^{2}$ de área sumergida.

\section{ANÁLISIS DE LOS RESULTADOS}

\subsection{Análisis de los resultados de las propiedades en estado fresco}

En todos los casos de $\mathrm{R} \mathrm{a/c}$ aumenta a medida que aumenta la relación aglomerante/agregado.

Las dosificaciones con los suelos A, B y C mantienen un crecimiento estable y muy parecido en el mismo orden, independientemente del tipo de cemento empleado, lo cual se corresponde con la cantidad de finos de los suelos, ya que el suelo $C$ tiene mayor cantidad de finos que el $B$ y éste más que el $\mathrm{A}$ y el suelo $\mathrm{D}$ es un suelo arcilloso el cual necesita mayor cantidad de agua en su mezclado para obtener igual fluidez en el mortero.

El suelo D también fue empleado en morteros de cal y morteros bastardos, debido a que como es un suelo arcilloso siempre es recomendado el trabajar con cales, ya que, en general, tienen mejor comportamiento al reaccionar los silicatos y aluminatos de los suelos con la cal formando silicatos cálcicos hidratados, mientras que en los suelos arenosos sin arcilla, la reacción de la cal es un proceso de carbonatacion de la cal en presencia del $\mathrm{CO}_{2}$ del aire, formándose, por una reacción muy lenta, carbonato de calcio.

La Tabla 7 se corresponde con los morteros bastardos. A medida que aumenta el hidrato de calcio en la dosificación hay un aumento de la relación agua/cemento.

\subsection{Análisis de los resultados de las propiedades en estado endurecido}

En las Tablas 3 y 4 se muestra el comportamiento de las resistencias a flexión y compresión a través del tiempo desde los 7 días hasta los 365 días.

En todos los casos hay un ascenso de las resistencias con el tiempo (rápido hasta los 90 días) y después el crecimiento se hace más lento, aunque siempre en ascenso.

La Tabla 5 recoge datos comparativos de resistencias de los morteros con los cementos P-350 y PP-250. En todos los casos la resistencia a la compresión disminuye cuando aumenta la relación aglomerante/agregado. Las mayores resistencias se alcanzan con el suelo A, que es un suelo gravoso arenoso y las menores con el suelo $\mathrm{D}$, que es una tierra arcillosa, lo cual se corresponde con la bibliografía, ya que, para los suelos arcillosos, se recomienda el empleo de mayor cantidad de cemento que para los suelos arenosos y gravosos, para obtener igual resultado.

La Tabla 6 muestra los resultados de los morteros de calsuelo D. Las resistencias a la compresión disminuyen a medida que aumenta la relación aglomerante-crudo pero el descenso es poco pronunciado, oscilando entre valores de 2,59 y 1,58 MP a los 28 días. Estos valores de 
TABLA 3

Morteros de albañilería. Resistencia a flexión y compresión (MPa). Fluidez $110 \pm 10 \mathrm{~mm}$

\begin{tabular}{|c|c|c|c|c|c|c|c|c|c|c|c|c|}
\hline \multirow{2}{*}{$\begin{array}{l}\text { Tipo } \\
\text { de } \\
\text { Suelo }\end{array}$} & \multirow{2}{*}{$\begin{array}{l}\text { Dosif. } \\
C+S\end{array}$} & \multirow[b]{2}{*}{$\mathrm{a} / \mathrm{c}$} & \multicolumn{2}{|c|}{$7 d$} & \multicolumn{2}{|c|}{$28 d$} & \multicolumn{2}{|c|}{$90 d$} & \multicolumn{2}{|c|}{$210 d$} & \multicolumn{2}{|c|}{$365 d$} \\
\hline & & & $\mathrm{Rf}$ & Rc & $R f$ & Rc & $\mathrm{Rf}$ & Rc & Rf & Rc & $\mathrm{Rf}$ & Rc \\
\hline \multirow{3}{*}{ A } & $1: 5$ & 1,33 & 0,94 & 4,29 & 2,25 & 6,15 & 3,55 & 10,47 & 4,01 & 11,62 & $4, ' 05$ & 11,73 \\
\hline & $1: 7$ & 1,78 & 0.47 & 2,35 & 1,77 & 4,33 & 2,37 & 7,16 & 2,65 & 7,77 & 2,66 & 8,42 \\
\hline & $1: 9$ & 2,09 & 0,45 & 1,77 & 1,15 & 3,27 & 1,48 & 4,45 & 1,51 & 5,42 & 1,53 & 6,22 \\
\hline \multirow{3}{*}{ B } & $1: 5$ & 1,39 & 1,21 & 2,89 & 2,12 & 5,13 & 3,27 & 10,27 & 4,00 & 10,30 & 4,02 & 11,56 \\
\hline & $1: 7$ & 1,85 & 0,68 & 2,02 & 1,40 & 3,93 & 2,61 & 6,30 & 2,64 & 7,32 & 3,04 & 8,70 \\
\hline & $1: 9$ & 2,20 & 0,55 & 1,61 & 1,09 & 2,89 & 1,30 & 3,53 & 1,90 & 5,88 & 1,93 & 5,91 \\
\hline \multirow{3}{*}{ C } & $1: 5$ & 1,47 & 0,70 & 2,59 & 1,81 & 4,01 & 2,00 & 5,26 & 2,05 & 5,40 & 2,34 & 7,56 \\
\hline & $1: 7$ & 1,96 & 0,60 & 2,09 & 1,29 & 3,21 & 1,44 & 4,04 & 1,54 & 4,33 & 1,76 & 4,79 \\
\hline & $1: 9$ & 2,35 & 0,56 & 1,65 & 0,88 & 2,79 & 0,88 & 3,44 & 0.91 & 3,66 & 1,08 & 4,36 \\
\hline
\end{tabular}

$\mathrm{C}=$ Cemento $\mathrm{P}-350$

TABLA 4

Morteros de albañilería. Resistencia a flexión y compresión (MPa). Fluidez $110 \pm 10 \mathrm{~mm}$

\begin{tabular}{|c|c|c|c|c|c|c|c|c|c|c|c|c|}
\hline \multirow[t]{2}{*}{$\begin{array}{l}\text { Tipo } \\
\text { de } \\
\text { Suelo }\end{array}$} & \multirow[t]{2}{*}{$\begin{array}{l}\text { Dosif. } \\
C+S\end{array}$} & \multirow[t]{2}{*}{$a / c$} & \multicolumn{2}{|c|}{$7 d$} & \multicolumn{2}{|c|}{$28 \mathrm{~d}$} & \multicolumn{2}{|c|}{$90 \mathrm{~d}$} & \multicolumn{2}{|c|}{$210 d$} & \multicolumn{2}{|c|}{$365 d$} \\
\hline & & & $R f$ & $\mathrm{Rc}$ & $\mathrm{Rf}$ & $\mathrm{Rc}$ & $\mathrm{Rf}$ & $\mathrm{Rc}$ & $R f$ & Rc & $R f$ & $\mathrm{Rc}$ \\
\hline \multirow{3}{*}{ A } & $1: 5$ & 1,39 & 0,70 & 3,36 & 2,34 & 7,94 & 2,53 & 9,35 & 2,62 & 10,33 & 3,22 & 10,54 \\
\hline & 1:7 & 1,82 & 0,47 & 1,58 & 1,91 & 3,70 & 1,91 & 4,85 & 1,94 & 5,99 & 2,00 & 6,55 \\
\hline & $1: 9$ & 2,15 & 0,22 & 1,49 & 1,28 & 3,31 & 1,40 & 4,11 & 1,42 & 4,64 & - & 4,66 \\
\hline \multirow{3}{*}{ B } & $1: 5$ & 1,47 & 1,11 & 4,02 & 2,69 & 7,31 & 3,45 & 10,34 & 3,60 & 10,99 & - & - \\
\hline & $1: 7$ & 1,97 & 0,47 & 2,28 & 1,77 & 4,61 & 1,99 & 4,77 & 2,02 & 4,96 & - & 5,50 \\
\hline & $1: 9$ & 2,31 & 0,23 & 1,04 & 0,89 & 3,07 & 1,28 & 3,26 & 1,35 & 3,47 & 1,46 & 4,22 \\
\hline \multirow{3}{*}{ C } & $1: 5$ & 1,61 & 0,69 & 3,25 & 1,16 & 4,53 & 1,67 & 5,34 & 1,84 & 6,85 & 2,89 & 7,55 \\
\hline & $1: 7$ & 2,12 & 0.45 & 1,52 & 0,89 & 3,20 & 1,30 & 3,34 & 1,32 & 3,79 & 1,58 & 4,76 \\
\hline & $1: 9$ & 2,55 & 0,21 & 1,05 & 0,55 & 2,75 & 0,88 & 2,99 & 0,91 & 3,03 & 0,94 & 3,53 \\
\hline
\end{tabular}

$\mathrm{C}=$ Cemento PP -320

resistencia son buenos para un mortero de cal, los cuales, por lo general, alcanzan muy baja resistencia a edades tempranas.

La Tabla 7 resumen los resultados obtenidos en los morteros bastardos. Estos morteros son los que mejor resistencia a la compresión alcanzan a los 28 días. Al igual que en las curvas anteriores, la resistencia a la compresión disminuye a medida que aumenta la relación aglomerante-crudo. No se obtiene mayor resistencia a medida que se aumenta la cal, manteniendo el cemento y suelo constante, aunque, obviamente, si se compara con los morteros cemento-suelo se aprecia, para similares relaciones, un aumento considerable de la misma, además la cal le aporta a los morteros mayor trabajabilidad y retención de agua, parámetros que son positivos por lo que respecta a los morteros de albañilería.

En las Tablas 5-7 aparecen los resultados de los ensayos de adherencia a tracción para diferentes tipos de morteros y para diferentes relaciones aglomerante-crudo. En todos los casos hay una disminución de la resistencia respecto 
TABLA 5

Morteros de albañilería. Resumen

\begin{tabular}{|c|c|c|c|c|c|c|c|}
\hline \multirow{2}{*}{$\begin{array}{l}\text { Tipo } \\
\text { De } \\
\text { Suelo }\end{array}$} & \multirow{2}{*}{$\begin{array}{l}\text { Dosif. } \\
C: S\end{array}$} & \multicolumn{2}{|c|}{$\mathrm{Rc}_{28 \mathrm{~d}}(\mathrm{MPa})$} & \multicolumn{2}{|c|}{ Radh. $28 \mathrm{~d}_{(\mathrm{MPa})}$} & \multicolumn{2}{|c|}{ Abs. Cap. $7 \mathrm{~d}\left(\mathrm{~g} / \mathrm{cm}^{2}\right)$} \\
\hline & & P350 & PP250 & P350 & PP250 & P350 & PP250 \\
\hline \multirow{3}{*}{ A } & 1,5 & 6,15 & 7,94 & 0,40 & 0,30 & 5,19 & 4,69 \\
\hline & 1,7 & 4,33 & 3,70 & 0,34 & 0,26 & 5,69 & 5,00 \\
\hline & 1,9 & 3,27 & 3,21 & 0,23 & 0,20 & 5,56 & 5,13 \\
\hline \multirow{3}{*}{ B } & 1,5 & 5,13 & 7,31 & 0,60 & 0,38 & 4,81 & 4,88 \\
\hline & 1,7 & 3,98 & 4,61 & 0,38 & 0,30 & 5,19 & 5,58 \\
\hline & 1,9 & 2,89 & 3,07 & 0,28 & 0,26 & 5,25 & 4,69 \\
\hline \multirow{3}{*}{ C } & 1,5 & 4,01 & 4,53 & 0,37 & 0,25 & 5,06 & 5,00 \\
\hline & 1,7 & 3,21 & 3,20 & 0,26 & 0,22 & 5,13 & 4,69 \\
\hline & 1,9 & 2,79 & 2,75 & 0,21 & 0,16 & 5,25 & 5,06 \\
\hline \multirow{3}{*}{$D$} & 1,5 & 4,13 & -- & 0,31 & -- & -- & -- \\
\hline & 1,7 & 2,70 & -- & 0,27 & -- & -- & -- \\
\hline & 1,9 & 1,98 & -- & 0,24 & - & -- & -- \\
\hline
\end{tabular}

TABLA 6

Morteros de albañilería. Resumen

\begin{tabular}{|cl|c|c|c|}
\hline \multicolumn{2}{|c|}{ Dosif. } & R a/c & $\mathrm{Rc}_{28 \mathrm{~d}}(\mathrm{MPa})$ & Radh. $_{\text {28d }}$ (MPa) \\
\hline H.C. S & 2 & 1,98 & 2,59 & 0,19 \\
\hline 1 & 2,5 & 2,37 & 2,15 & 0,16 \\
\hline 1 & 3 & 2,55 & 1,96 & 0,14 \\
\hline 1 & 3,5 & 2,59 & 1,84 & 0,09 \\
\hline 1 & 4 & 3,39 & 1,58 & - \\
\hline
\end{tabular}

$\mathrm{HC}=$ Hidrato de $\mathrm{Cal}(\mathrm{DPA}) ; \mathrm{S}=$ Suelo $\mathrm{D}$

TABLA 7

Morteros de albañilería. Resumen

\begin{tabular}{|ccc|c|c|c|}
\hline \multicolumn{3}{|c|}{ Dosif. } & $\mathrm{R} \mathrm{a/c}$ & $\mathrm{Rc}_{28 \mathrm{~d}}(\mathrm{MPa})$ & $\mathrm{Radh}_{28 \mathrm{~d}}(\mathrm{MPa})$ \\
\hline $\mathrm{C}: \mathrm{S}: \mathrm{HC}$ & 4 & 1 & 2,16 & 8,57 & 0,31 \\
\hline 1 & 5 & 1 & 2,36 & 8,35 & 0,22 \\
\hline 1 & 6 & 1 & 2,75 & 6,66 & 0,14 \\
\hline 1 & 7 & 1 & 3,02 & 6,47 & 0,14 \\
\hline 1 & 4 & 2 & 2,43 & 7,60 & 0,29 \\
\hline 1 & 5 & 2 & 2,63 & 7,09 & 0,27 \\
\hline 1 & 6 & 2 & 3,24 & 6,25 & 0,25 \\
\hline 1 & 7 & 2 & 3,38 & 5,56 & 0,16 \\
\hline
\end{tabular}

$\mathrm{C}=$ Cemento PP-350; $\mathrm{S}=$ Suelo $\mathrm{D} ; \mathrm{HC}=$ Hidrato de $\mathrm{Cal}$ (DPA) 
a la relación aglomerante-árido. Los mejores resultados se obtienen con el suelo B seguido del A, donde ambos suelos son ricos en gravas y arenas, pero a medida que los suelos van aumentando en finos, se observa un decrecimiento de la resistencia.

Con el hidrato de cal y el suelo D (Tabla 6) igualmente presenta disminución la resistencia respecto a la relación aglomerante-árido, aunque los resultados son buenos para ser realizados con morteros de cal. Este ensayo sólo se realizó con el suelo D, que es un suelo arcilloso.

En la Tabla 7 se muestra la resistencia a la adherencia contra relación aglomerante-agregado para diferentes dosificaciones, empleando cemento P350, hidrato de cal y el suelo D. Se aprecia una mejoría en la resistencia a la adherencia cuando se aumenta, en la dosificación, la cantidad de cal.

En la Tabla 5 aparecen los resultados de los ensayos de absorción capilar. Los valores obtenidos son relativamente altos si se comparan con los morteros tradicionales (cemento-cal-arena), que se mantienen aproximadamente alrededor del $3 \%$, aunque se conșidera que éste no es un factor determinante para los morteros. La absorción capilar está relacionada con la permeabilidad, porosidad y densidad del mortero, entre otros factores. A medida que un mortero es más denso, como consecuencia, es menos poroso y, por consiguiente, la absorción capilar debe disminuir, al igual que la permeabilidad.

Cuando se trabaja en paredes con elevada humedad es necesario el empleo de morteros permeables con el objeto de airear el muro y disminuir la humedad. Oliveira (6) plantea que una mayor porosidad, desde el punto de vista de conservación, de una restauración de edificios antiguos, es una virtud, ya que deja respirar a los muros. Este concepto bien se pudiera aplicar a este tipo de mortero.

\section{CONCLUSIONES}

Se caracterizó una serie de morteros de albañilería con suelo que abarcó dos tipos de cemento, un tipo de hidrato de cal y cuatro tipos de suelos (desde los arenosos hasta los arcillosos) obteniéndose resultados de resistencias mecánicas a flexión y compresión y adherencia a tracción perpendicular y ensayos de absorción capilar.

En todos los casos la relación agua/cemento aumenta, y las resistencias a flexión, compresión y adherencia disminuyen a medida que aumenta la relación aglomerante/ árido.

La relación agua/cemento en las dosificaciones con los suelos A, B y C mantienen un crecimiento estable, independientemente del cemento empleado, lo cual se corresponde con la cantidad de finos en los suelos, mientras que con el suelo $\mathrm{D}$, que es típicamente arcilloso, se necesita mayor cantidad de agua en el mezclado para obtener igual fluidez.

Las mayores resistencias a la compresión se alcanzan con los suelos A y B que son gravoso/arenoso, e incluso son ligeramente más altas con el empleo del cemento PP-250.

Las menores resistencias son con el suelo D.

En las dosificaciones con hidrato de cal se obtienen bajas resistencias mecánicas, pero similares a las obtenidas en morteros con el empleo de las arenas.

Las resistencias a la adherencia presentan similar evolución que las resistencias a la compresión, aunque los mejores resultados se obtienen con el suelo B.

En las dosificaciones bastardas (cemento-hidrato de calsuelo) se logran mejores resistencias a la adherencia, cuando se aumenta el contenido de hidrato de cal.

Los resultados del ensayo de adherencia es válido para ladrillos de suelo-cemento como substrato, donde se obtienen buenos resultados.

En general, los morteros de albañilería con suelo alcanzan valores de resistencia a compresión y adherencia similares a los morteros tradicionales de mortero-arena-hidrato de cal.

La absorción capilar fue mayor en, aproximadamente, un $2 \%$ que en los mortreros tradicionales.

\section{BIBLIOGRAFÍA}

(1) Salas, J. La Tierra. Material de Construcción. Monografia Nº385/386. Instituto Eduardo Torroja. $1^{\text {a }}$ Ed.. 1987. España. Págs. $11-12$.

(2) Navarro,N. y otros. Suelo-Cemento. Fundamentos para la aplicación en Cuba. Impreso en Cuba, 1991. 208 Págs.

(3) NC52-79:93. Morteros de Albañilería. Parte 1. Diseño y Caracterización, Código de Práctica para la Construcción. Vig. enero 1993. 8 Págs.

(4) NC54-207:80. Cemento. Ensayos físico-mecánicos. Vig. enero 1982. 23 Págs.

(5) Álvarez J.L. La adherencia en los morteros. Normas y resultados. Memorias III Conferencia Científico-Técnica de la Construcción. Cuba, abril 1998.

(6) Oliveira, M. y otros. Argamasas bastardos. Origen y propiedades. I Simposio Brasileiro de Tecnología das Argamassas, agosto 1995. Págs. 43-52. 